EPSC Abstracts

Vol. 14, EPSC2020-636, 2020

https://doi.org/10.5194/epsc2020-636

Europlanet Science Congress 2020

(C) Author(s) 2021. This work is distributed under

the Creative Commons Attribution 4.0 License.

\title{
The activity of the Jupiter co-orbital comet P/2019 LD2 (ATLAS) observed with the $10.4 \mathrm{~m}$ GTC.
}

\author{
Javier Licandro ${ }^{1}$, Julia de Leon ${ }^{1}$, Fernando Moreno ${ }^{2}$, Carlos de la Fuente Marcos ${ }^{3}$, Raul de la Fuente \\ Marcos $^{3}$, Antonio Cabrera Lavers ${ }^{5}$, Luisa Lara ${ }^{2}$, Noemi Pinilla-Alonso ${ }^{6}$, Mario de Pra ${ }^{6}$, Ana Carolina de \\ Souza-Feliciano ${ }^{6}$, and Stefan Geier ${ }^{5}$ \\ ${ }^{1}$ Instituto de Astrofisica de Canarias, Research, La Laguna, Tenerife, Spain (jlicandr@iac.es) \\ ${ }^{2}$ Instituto de Astrofisica de Andalucia, CSIC, Glorieta de la Astronomia s/n, 18008 Granada, Spain \\ ${ }^{3}$ Universidad Complutense de Madrid, Ciudad Universitaria, E-28040 Madrid, Spain \\ ${ }^{5}$ GRANTECAN, Cuesta de San Jose s/n, E-38712 Breña Baja, La Palma, Spain \\ ${ }^{6}$ Florida Space Institute, 12354 Research Parkway Partnership 1 Building, Suite 214 Orlando, FL 32826-0650, USA
}

Introduction: Comet P/2019 LD2 (ATLAS) was discovered early June 2019 as a faint asteroidal object and initially classified as a Jupiter Trojan. In depth inspection of images obtained during 2019 revealed that the object is active. No signs of cometary-like activity have been detected on any Jupiter Trojans until now, despite it is widely accepted that they are captured objects from the outer solar system. For that reason, we scheduled P/2019 LD2 observations with the world's largest optical telescope, the $10.4 \mathrm{~m}$ Gran Telecopio CANARIAS (GTC) to study its nature as soon as it became visible in May 2020

In the meantime, Kareta et al. (2020) showed that the comet had experienced a close encounter with Jupiter on February 17, 2017 at $0.092 \mathrm{AU}$, well inside the Hill radius of the planet, $0.338 \mathrm{AU}$. They conclude that it is a recently captured Centaur, not a Jupiter Trojan.

Even if P/2019 LD2 is not the first active Jupiter Trojan, it is a very interesting object that could help to better understand the transition from Centaur to JFC. Its orbit just beyond Jupiter and its activity are evocative of 29P/Schwassmann-Wachmann, an object considered as a prototypical "gateway" between the Centaurs and JFCs by Sarid et al. (2019).

Observations: We present the observations of P/2019 LD2 (ATLAS) obtained on 2020 May 16 and 17 using the OSIRIS camera-spectrograph of the $10.4 \mathrm{~m}$ GTC.

On May 16 we obtained images, using the Sloan $\mathrm{g}^{\prime}, \mathrm{r}^{\prime}, \mathrm{i}^{\prime}, \mathrm{z}^{\prime}$ filters that were used to characterize its overall level of cometary activity using a Monte Carlo dust tail fitting code as described in various papers (see, e.g. Moreno et al. 2016, 2017, and references therein). The comet presents a conspicuous coma and tail as seen Fig. 1.

We also obtained two visible spectra of P/2019 LD2 on May 17 with the aim of looking for signatures of the typical gas species observed in comets. Each individual spectra consisted of an exposure of 600 seconds using the R300B grism and the $1.49^{\prime \prime}$ slit width, covering a wavelength range from 

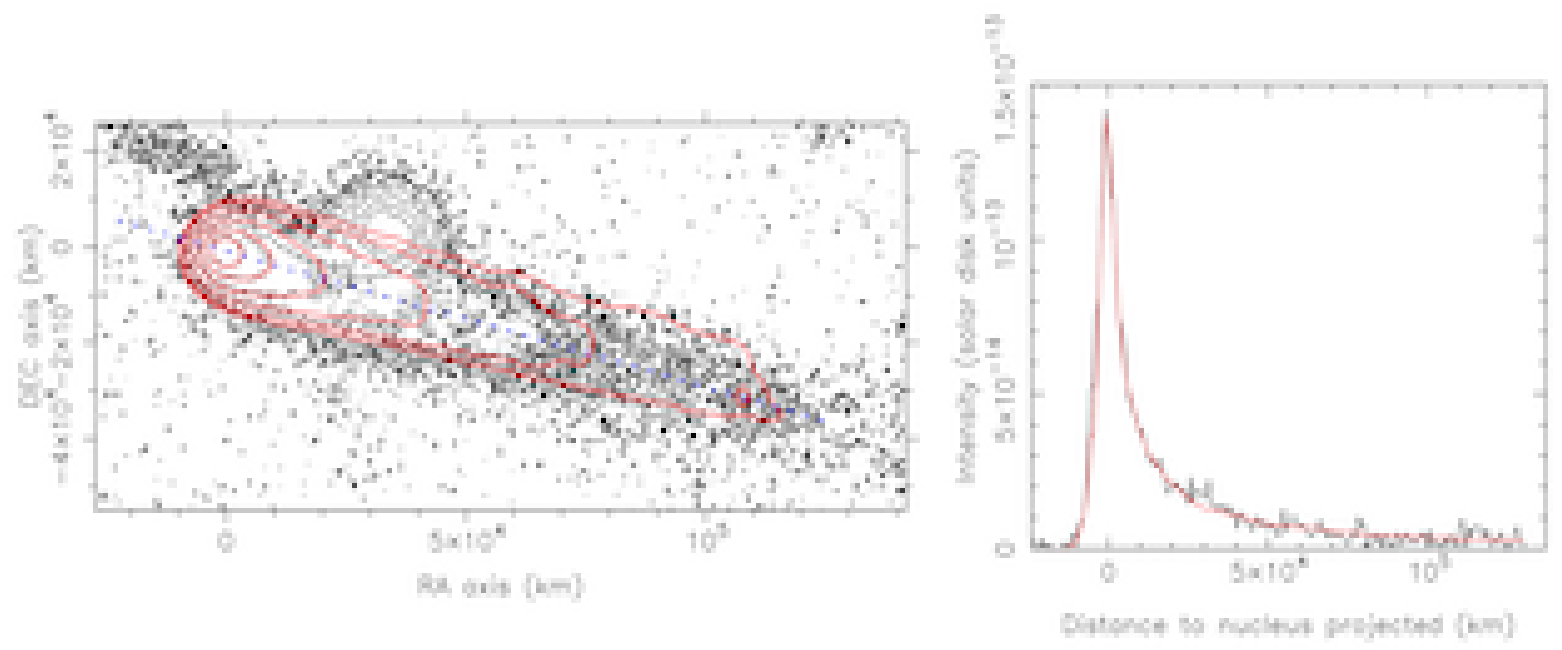

Fig. 1 Left panel: A comparison of the observed (black contours) and modeled (red contours) tail brightness isophotes. The innermost contour corresponds to $8 \times 10^{-14}$ solar disk units, and the brightness decrease in factors of two outwards. The images are rotated to the conventional Northup, East-to-the-left orientation. The $\mathrm{x}$ - and $\mathrm{y}$-axis are labeled in $\mathrm{km}$ projected on the sky at the object distance. Right panel: A comparison of observed (black line) and modeled (red line) tail brightness along the direction described by the blue dotted line in the left panel.

Past, present and future dynamical evolution: The assessment of the dynamical evolution of $\mathrm{P} / 2019$ LD2 requires the analysis of an extensive sample of $\mathrm{N}$-body simulations. In this work, we have used the approach discussed in de la Fuente Marcos \& de la Fuente Marcos (2019) and Licandro et al. (2019) to study the past, present and future evolution.

Results: Our results can be summarized as follows:

(i) P/2019 LD2 shows a conspicuous coma and tail with a longitude $>1^{\prime}$.

(ii) There is no evidence of $\mathrm{CN}, \mathrm{C} 2$ or $\mathrm{C} 3$ emission within the 3- $\sigma$ level in the comet spectrum. In particular there are no signs of the $\mathrm{CN}(0-0)$ emission at $3880 \mathrm{~A} \square$ that it is usually the strongest emission observed in comets.

(iii) According to our model, the dust emission of P/2019 LD2 can be described by a Gaussian with a FWHM $=180$ days, a maximum (dM/dt)0 $=50 \mathrm{~kg} \mathrm{~s}^{-1}$ attained on 2019 November 27 ( $\mathrm{t}=170$ days from the observations) that then decreases again, with a current (2020 May 16) dust loss rate of 5 $\mathrm{kg} \mathrm{s}^{-1}$. This implies a total dust mass loss of $9 \times 10^{8} \mathrm{~kg}$ since the start of the dust emission, and almost no dust emission when observed in 2018.

(v) The origin of activity is most likely linked to a thermally driven process, likely associated to sublimation of crystalline water ice and clathrates

(vi) The nucleus corresponds to a km-size object (with radius between 1.5 and $3.5 \mathrm{~km}$ ), in the sizerange of the JFCs.

(vii) P/2019 LD2 is now an ephemeral co-orbital of Jupiter, following what looks like a short arc of a 
quasi-satellite cycle that started in 2017 and will end in 2028. It will experience a very close encounter with Jupiter at $\square 10$ Jovian radii on January 18, 2063. If it survive the close approach, its probability of escaping the solar system during the next $0.5 \mathrm{Myr}$ is $0.48 \pm 0.02$

(ix) The origin of P/2019 LD2 is still an open question. The probability of this comet having been captured from interstellar space during the last $0.5 \mathrm{Myr}$ is $0.50 \pm 0.03,0.79 \pm 0.06$ during the last 2 Myr and to $0.89 \pm 0.07$ for $4 \mathrm{Myr}$, suggesting that P/2019 LD2 can be a captured interstellar comet. Anyhow, a very close encounter with one of the giant planets of a former member of the scattered disk may have produced a fragmentation event induced by the planetary tidal force that was eventually able to form the observed, present-day, P/2019 LD2 .

\section{References}

de la Fuente Marcos, C., de la Fuente Marcos, R., Licandro, J., Serra-Ricart, M., \& Cabrera-Lavers, A. 2019, Research Notes of the American Astronomical

Society, 3, 143

Kareta, T., Volk, K., Noonan, J. W., et al. 2020, Research Notes of the American Astronomical Society, 4, 74

Licandro, J., de la Fuente Marcos, C., de la Fuente Marcos, R., et al. 2019, A\&A, Moreno, F., Pozuelos, F. J., Novakovic', B., et al. 2017, ApJ, 837, L3

Moreno, F., Snodgrass, C., Hainaut, O., et al. 2016, A\&A, 587, A155

Sarid, G., Volk, K., Steckloff, J. K., et al. 2019, ApJ, 883, L25 